\author{
Aynur Özge \\ Cengiz Özge \\ Hakan Kaleagasi \\ Osman Özgür Yalin \\ Özgür Ünal \\ Eylem S. Özgür
}

\section{Headache in patients with chronic obstructive pulmonary disease: effects of chronic hypoxaemia}

Received: 28 September 2005

Accepted in revised form: 11 December 2005

Published online: 17 January 2006

A. Özge • H. Kaleagasi • O.Ö. Yalin • Ö. Ünal Department of Neurology

Mersin University School of Medicine

Mersin, Turkey

C. Özge • E.S. Özgür

Department of Chest Disease

Mersin University School of Medicine

Mersin, Turkey

A. Özge (ه)

Fatih mah. Istanbul Evleri C Blok D:5

Mezitli 33170, Mersin, Turkey

e-mail: aozge@mersin.edu.tr

Tel.: +90-324-359-8904

Fax: +90-324-359-4336

\begin{abstract}
The frequency and characteristics of headache in patients with chronic obstructive pulmonary disease (COPD) are not clear and there are only a few studies that have assessed the relationship between chronic hypoxaemia and headache. We performed this study in order to evaluate the frequency and characteristics of headache in COPD patients. A total of 119 patients, with a mean age of $63.4 \pm 8.2$ years, diagnosed with moderate or severe stable COPD were included in the study. Overall $31.9 \%$ of the patients complained of headache and $45.4 \%$ were reported to have sleep disorders. There were significant effects of family
\end{abstract}

\begin{abstract}
history of COPD, having other systemic disorders or sleep disorders (snoring, bruxism, restless leg syndrome, etc.) and laboratory data of chronic hypoxaemia and airway obstruction on headache co-morbidity. In conclusion, possibly being a specific subtype of elderly headache, headache in patients with moderate or severe COPD is a common problem and future studies are needed to obtain more knowledge about its pathophysiological and clinical basis.
\end{abstract}

Keywords Chronic obstructive pulmonary disease $\cdot$ Headache . Chronic hypoxaemia $\cdot$ Sleep disorders $\cdot$ Pulmonary function tests

\section{Introduction}

Chronic obstructive pulmonary disease (COPD) is a disease state characterised by airflow limitation that is not fully reversible. The airflow limitation is usually both progressive and associated with an abnormal inflammation of the lungs to noxious particles or gases, which leads to hypoxaemia and hypercarbia due to a poor ratio of ventilation to perfusion in the lung parenchyma [1].

ICHD-II criteria reported that although morning headache is significantly more common in patients with sleep apnoea than in the general population, headache pre- sent upon awakening is a non-specific symptom that occurs in a variety of primary or secondary headache disorders, in sleep-related respiratory disorders other than sleep apnoea (e.g., Pickwickian syndrome, COPD), and in other primary sleep disorders such as periodic leg movements during sleep. The criteria point out that a definitive diagnosis of sleep apnoea headache (10.1.3) requires overnight polysomnography. They also report that it is unclear whether the mechanism of sleep apnoea headache is related to hypoxia, hypercapnia or disturbance in sleep [2].

However, diagnostic criteria for hypnic headache, which occurs exclusively during sleep and usually begins after the age of 60 years, include the following: headache 
occurs only during sleep and awakens the patient, is not associated with autonomic symptoms, and is associated with no more than one of the following: nausea, photophobia or phonophobia. Hypnic headache diagnosis requires two of the following features: occurs at least 15 times per month; lasts more than 15 minutes; onset occurs after age $50[3,4]$. Hypnic headache was the main diagnosis in $0.09 \%$ of all headache patients and notably in $1.4 \%$ of geriatric patients (above the age of 65 years) seen in Headache Centre reports [3].

In adults, a few simple questions can direct the history to identify patients 'at risk' for sleep apnoea and other sleep disorders. These are inquiries concerning: the restorative nature of the patient's sleep; excessive daytime sleepiness, tiredness or fatigue; the presence of habitual snoring; and whether the total sleep time is sufficient. They can be extremely revealing. This questionnaire has been named as "pneumonic REST" and can help the clinician remember these four key questions in screening history [4].

Sleep-related disorders are long-recognised conditions. However, the clinical importance of these conditions and the relationship between them and specific conditions such as COPD are not well understood. Common sleep disorders that have been associated with headache include insomnia, obstructive sleep apnoea (OSA) and restless legs syndrome/periodic limb movement disorder [4-6].

The objectives and hypotheses for our study were derived from previous research and from a review of the nature of COPD. Although sleep-related headache is commonly well understood, clinical characteristics of headache attributed to chronic hypoxia or hypercapnia have not been described yet. We performed this cross-sectional clinicalbased study in order to determine the frequency and characteristics of headache in patients with moderate or severe COPD, which was an important cause of chronic hypoxaemia and hypercarbia, and to evaluate the importance of additional sleep disturbance of these patients.

\section{Patients and methods}

In this study we investigated 119 patients with moderate or severe COPD according to the criteria of the Global Initiative for Chronic Obstructive Lung Disease (GOLD). Patients with $\mathrm{FEV}_{1}$ levels lower than 50\% were included in this study [1]. All subjects gave written informed consent for the study protocol and detailed explanations were given according to the Helsinki declaration.

Inclusion criteria for the study were as follows: strict diagnosis of moderate or severe COPD according to the GOLD criteria; free of psychotropic drugs for at least 2 weeks prior to the study to obtain a high degree of cooperation; no history of neurological diseases (epilepsy, stroke, acute head injury, dementia, toxic chemical exposure, nocturnal seizures, caffeine withdrawal, drug abuse etc); no concomitant severe or chronic illnesses (hepatic failure, chronic renal failure, out of control diabetes mellitus, other endocrine disorders, chronic infections, etc.); no history of headache attributed to chronic post-traumatic headache or patients with aphasia or other speech alterations who could not describe their headache and sleep disorders, no radiographic chest features of interstitial lung disease.

Detailed physical examinations were performed by the same pulmonary disease specialist (C. Örge) and full neurological examinations as well as headache diagnosis according to ICHD-II criteria [2] were made by the same neurologist (A. Örge). A detailed history of headache was obtained from all of the patients with a structured interview (Ö.Ümal, O.Ö. Yalin). The severity of headache was evaluated with a visual analogue scale (VAS) in the range of $0-10$ [7]. Other headache characteristics including frequency, quality, trigger factors, relieving factors, waking up with headache from sleep and associated symptoms such as nausea, vomiting, photophobia and phonophobia were also recorded. Detailed neurologic examinations as well as cerebral computed tomography (CT) were performed to exclude secondary causes of headache in these patients. If CT imaging was reported as suspicious or the patients had any signs or symptoms related to posterior fossa abnormalities (such as cerebellar signs), then magnetic resonance imaging (MRI) was performed for the evaluation as well.

Chest radiographs, respiratory function tests and arterial blood gas analyses were performed for all subjects in order to make a differential diagnosis. The electrocardiography, haemogram, serum electrolytes, fasting serum glucose, haemoglobin $\mathrm{A}_{1} \mathrm{C}$ ratio, renal functions, $\mathrm{CPK}$, vitamin $\mathrm{B}_{12}$ and folic acid levels of the patients were also recorded in order to exclude concurrent risk factors for headache evaluations. Sleep disorders were evaluated using pneumonic REST [4] and the specific subtypes have been determined where possible. We could not perform polysomnographic records of the patients because of the absence of the necessary technical support. The patients who had clinical and supportive laboratory features of sleep apnoea syndrome (i.e., high BMI score, positive features of pneumonic REST, normal CT scan for brain, positive history of sleep apnoea from their partners, etc.) were classified as "possible sleep apnoea syndrome".

The respiratory function tests were performed using a spirometry device (Sensormedics Vmax 22, CA, USA) and a computer. Patients were required to sit in an upright position during the tests. We calculated the forced expiratory volume in $1 \mathrm{~s}\left(\mathrm{FEV}_{1}\right)$, the forced vital capacity $(\mathrm{FVC})$, and $\mathrm{FEV}_{1} / \mathrm{FVC}$ ratio from maximal expiratory manoeuvres. Data were expressed as a percentage of the predicted standardised values according to European Respiratory Society (ERS) criteria [8]. Acceptable recordings were obtained from each manoeuvre and the highest values were used for further analysis $[9,10]$. The $\mathrm{pH}$, arterial oxygen tension $\left(\mathrm{PaO}_{2}\right)$, arterial carbon dioxide tension $\left(\mathrm{PaCO}_{2}\right)$ and $\mathrm{HCO}_{3}$ levels were analysed in radial artery blood samples with the patients in a resting position.

The results of descriptive analyses were tested and found to show normal distribution, thus data were given as the means and standard deviations. Parametric data were compared using unpaired $t$-test and non-parametric data were compared using the $\chi^{2}$ or Fisher's exact tests. The correlations between the headache parameters, pulmonary function tests and the levels of 
arterial blood gases were determined using appropriate statistics. Binary logistic regression analysis was used to determine the predictive factors for headache and sleep disturbance subtypes. Significant differences (two-tailed $p$ ) less than 0.05 were regarded as significant.

\section{Results}

The mean age of our 119 COPD patients was 64.2 \pm 9.1 years (range 42-74 years) and males were predominant (87.4\%, 104 patients). Demographic features of the study groups have been shown in Table 1. The COPD group was composed of patients having severe airway obstruction and severe arterial hypoxaemia with hypercarbia as shown in Table 1. Among the COPD group, 100 patients (84.0\%) were smokers, and the rest $(16.0 \%)$ were non-smokers. Patients underwent standardised management for their COPD and co-morbid systemic diseases, such as hypertension, according to the study protocol. The main drugs they were given were long-acting inhaled $\beta-2$ agonists, inhaled anticholinergic agents, inhaled glucocorticosteroids and sustained release theophylline. None of them received continuous oxygen therapy. Among the study subjects 35 patients $(29.4 \%)$ reported having a family history of COPD.

Among the subjects that underwent our study protocol, $38(31.9 \%)$ subjects reported chronic headache complaints. Seven of them (18.4\%) were female and the others $(81.6 \%)$ were male. According to new ICHD-II criteria they were classified as follows: typical aura with migraine headache ( 2 patients), migraine without aura (4 patients), frequent episodic tension-type headache (7 patients), infrequent episodic tension-type headache (5 patients), chronic tension-type headache (10 patients), benign cough headache ( 2 patients), hypnic headache (1 patient), headache related to arterial hypertension (5 patients), symptomatic cough headache (1 patient) and headache related to intracranial lesion (1 patient) (Fig. 1). Apart from one chronic subdural haematoma and one Chiari type one abnormality, none of them showed important neuroradiological abnormality during routine investigations. Six of the patients reported two types of headache together (hypnic headache plus chronic tension-type headache and migraine plus chronic tension-type headache). Their first diagnoses have been used in the previous breakdown.

The COPD patients with headache reported a mean headache duration of 7.6 \pm 7.5 years (range 3 months to 20

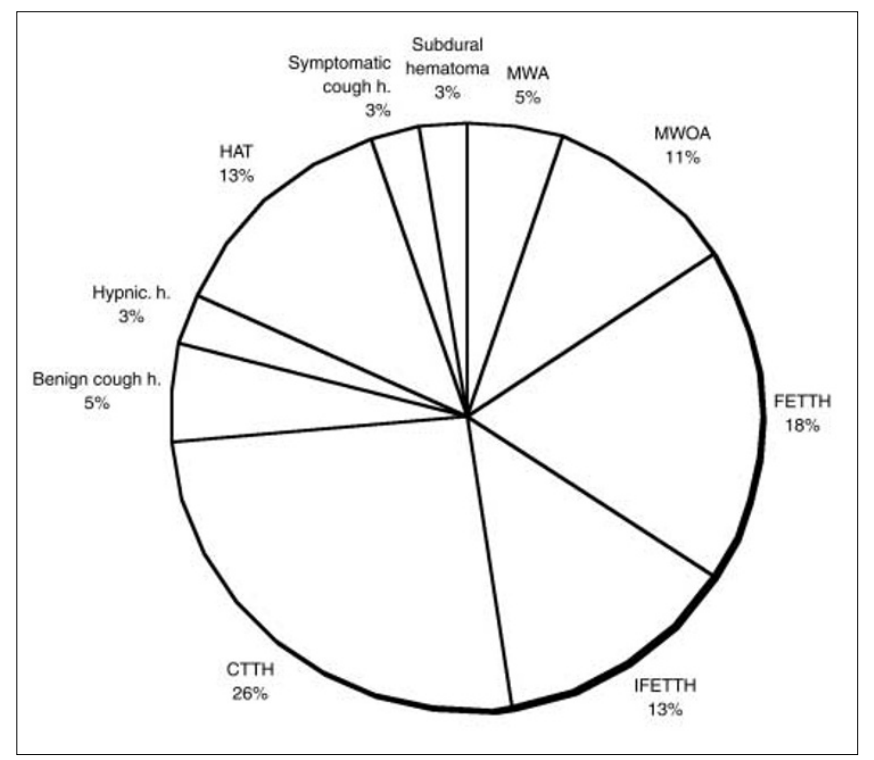

Fig. 1 Headache subtypes of COPD patients (MWA, typical aura with migraine; $M W O A$, migraine without aura; FETTH, frequent episodic tension-type headache; IFETTH, infrequent episodic tension-type headache; $C T T H$, chronic tension-type headache; $H A T$, headache related to arterial hypertension)

Table 1 Demographic characteristics of the patients with COPD according to headache $(n=119)$. Data are mean \pm SD unless otherwise indicated

\begin{tabular}{llll}
\hline Parameters & Headache $(n=38)$ & Headache free $(n=81)$ & $p$ \\
\hline Age, years & $63.5 \pm 11.8$ & $64.5 \pm 7.5$ & 0.588 \\
Gender, female(\%)/male(\%) & $7(18.4) / 31(81.6)$ & $8(9.9) / 73(90.1)$ & 0.190 \\
Duration of COPD, years & $8.1 \pm 7.0$ & $8.6 \pm 7.5$ & 0.719 \\
Smoking, packed/years & $35.6 \pm 24.1$ & $45.1 \pm 30.9$ & 0.100 \\
pH & $7.40 \pm 0.04$ & $7.39 \pm 0.02$ & 0.225 \\
Partial pressure $\mathrm{O}_{2}, \mathrm{mmHg}$ & $76.7 \pm 14.2$ & $67.2 \pm 12.1$ & 0.000 \\
Partial pressure $\mathrm{CO}_{2}, \mathrm{mmHg}$ & $40.9 \pm 9.7$ & $41.4 \pm 6.7$ & 0.781 \\
$\mathrm{HCO}_{3}$ & $24.0 \pm 6.3$ & $25.1 \pm 4.3$ & 0.263 \\
FEV,$\%$ & $54.5 \pm 14.9$ & $45.3 \pm 17.8$ & 0.006 \\
$\mathrm{FVC}_{1} \%$ & $74.8 \pm 19.0$ & $69.6 \pm 21.7$ & 0.203 \\
$\mathrm{FEV}_{1} / \mathrm{FVC}$ ratio & $56.1 \pm 7.2$ & $50.7 \pm 9.4$ & 0.002 \\
\hline
\end{tabular}


years). According to personal headache diaries, the frequency of the attacks was $10.3 \pm 9.8$ days/month, the mean duration of attacks was $6.8 \pm 6.5 \mathrm{~h} /$ day and the mean severity of attacks was $5.5 \pm 1.3$ according to the VAS scale. Twelve of the patients $(31.6 \%)$ described their headache as throbbing or stabbing in quality, while others (68.4\%) reported having headache pressing in quality. Among the associated symptoms, photophobia or phonophobia (6 patients, $15.8 \%$ ) and vomiting (11 patients, $28.9 \%)$ were reported at different frequencies. Six of the patients $(15.8 \%)$ reported aggravation of headache attacks by physical activity and 23 of the patients $(60.6 \%)$ by stress. They commonly reported headache attacks being relieved by resting (19 patients, $50.0 \%$ ) or sleeping (8 patients, $21.1 \%)$. Fifteen of the COPD subjects $(30.5 \%)$ reported only morning headaches.

Sleep disorders were reported by 54 patients with COPD $(45.4 \%)$ and 21 of them $(38.9 \%)$ reported headache also. The mean duration of sleep disorders of patients was 4.1 \pm 3.4 years. The details of COPD patients with sleep disorders are summarised in Table 2.

Pearson and Spearman correlation analysis showed some important correlations between headache and the following variables: FEV1\% (CC: -0.249, $p=0.006)$, FEV1/FVC ratio (CC: $-0.276, p=0.002$ ), $\mathrm{PaO}_{2}$ levels (CC: $-0.328, p=0.000)$, negative family history of COPD (CC: $0.270, p=0.003)$, possible sleep apnoea syndrome (CC: $0.225, p=0.024)$, snoring (CC: $0.385, p=0.000$ ), fatigue in mornings (CC: $0.300, p=0.001$ ), a history of bruxism (CC: $0.248, p=0.007$ ) or restless leg syndrome (CC: 0.287 , $p=0.002$ ). These results suggested the positive effects of chronic hypoxaemia and associated sleep disorders on the headache symptoms of the COPD patients.

Binary logistic regression analysis (Enter method) showed a general significant abnormality in this model $(p=0.001$, Exp B: 1.97). This model showed that negative family history of COPD, co-morbid medical disorders of the patients (hypertension or compensate diabetes mellitus), $\mathrm{FEV}_{1} \%$ levels, $\mathrm{FVC} \%$ levels, $\mathrm{FEV}_{1} / \mathrm{FVC}$ ratios,
$\mathrm{PaO}_{2}$ levels, possible sleep apnoea syndrome, snoring, fatigue in mornings, a history of bruxism or restless leg syndrome had important effects on headache with different scores (Table 3). Regression analysis results suggested that the important effects of chronic hypoxaemia and sleep disorders on headache complaints were independent from specific headache subtypes. Binary logistic regression analysis of sleep disorders and their subtypes showed important effects of age, duration of COPD, FEV $1 \%$ levels, FVC\% levels, FEV1/FVC ratios, $\mathrm{PaO}_{2}$ and $\mathrm{PaCO}_{2}$ levels with different scores (Table 3).

\section{Discussion}

We investigated moderate or severe COPD patients, as a rare investigated subtype of headache, in order to determine specific effects of chronic hypoxaemia on headache or sleep disturbance and additionally we aimed to evaluate the effects of sleep disturbance on this condition. We showed $31.9 \%$ of COPD subjects had headache with different subtypes; $45.4 \%$ reported sleep disturbances and 21 of them $(38.9 \%)$ reported headache also.

The new ICHD-II classification provides specific criteria for specific subtypes of homeostasis disturbances such as sleep apnoea or high altitude syndrome, but the effects of chronic hypoxaemia or hypercarbia were not clear [2]. As a cause of chronic hypoxaemia or hypercarbia, COPD is a common health problem in our country and worldwide [1]. Generally specific headache diagnosis can easily be made by using the new ICHD-II classification criteria, but sometimes patients do not fulfil the criteria (i.e., discordance between headache severity and frequency or headache location, and associated features). In this context a discussion on conditions in which the headache attributed to chronic hypoxaemia can be opened. At this point we do not know whether it is a specific headache type or these symptoms are additional features

Table 2 Demographic characteristics of the patients with COPD according to sleep disorders. Data are mean \pm SD unless otherwise indicated

\begin{tabular}{llll}
\hline Parameters & Sleep disorders $(n=54)$ & No sleep disorder $(n=65)$ & $p$ \\
\hline Age, years & $64.8 \pm 9.9$ & $63.5 \pm 8.0$ & 0.420 \\
Gender, female $(\%) /$ male $(\%)$ & $11(20.4) / 43(79.6)$ & $4(6.2) / 61(93.8)$ & 0.020 \\
pH & $7.40 \pm 0.02$ & $7.39 \pm 0.03$ & 0.225 \\
Partial pressure $\mathrm{O}_{2}, \mathrm{mmHg}$ & $71.0 \pm 12.7$ & $69.4 \pm 14.6$ & 0.519 \\
Partial pressure $\mathrm{CO}_{2}, \mathrm{mmHg}$ & $40.4 \pm 6.7$ & $41.5 \pm 8.9$ & 0.678 \\
$\mathrm{HCO}_{3}$ & $24.9 \pm 4.5$ & $24.5 \pm 5.6$ & 0.665 \\
$\mathrm{FEV}_{1}, \%$ & $44.0 \pm 16.4$ & $53.3 \pm 17.3$ & 0.004 \\
$\mathrm{FVC}, \%$ & $67.9 \pm 21.3$ & $75.3 \pm 19.9$ & 0.054 \\
FEV & FVC ratio & $55.7 \pm 8.1$ & 0.000 \\
\hline
\end{tabular}


Table 3 The important factors affecting headache and sleep disorders

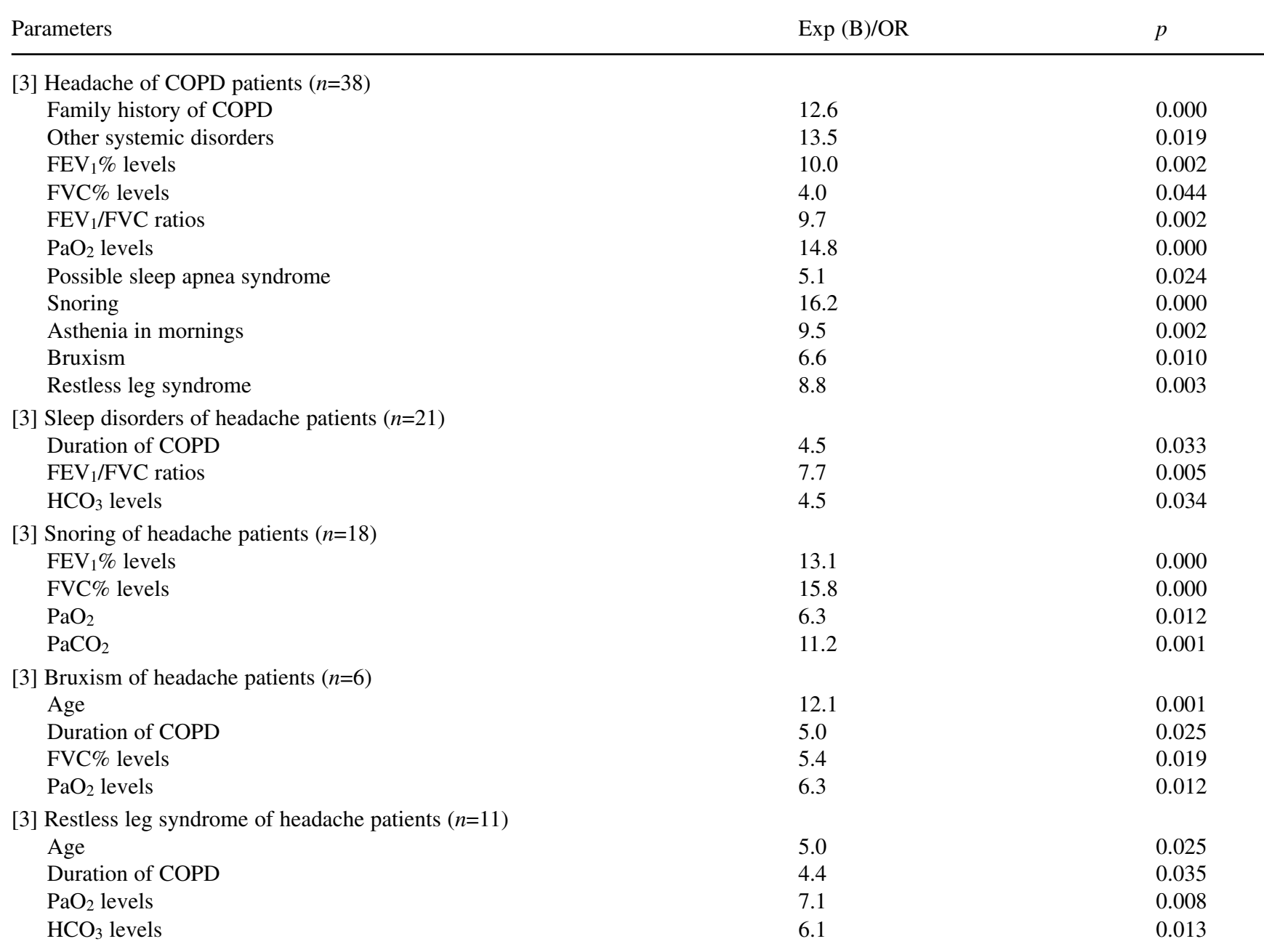

of elderly headache. It is also not obvious whether the additional sleep disorders have specific meaning for headache or not.

The International Committee of Sleep Disorders (ICSD) includes a diagnosis for 'sleep-related headache', and it is recommended that an ICHD-II headache diagnosis should be stated in conjunction with the ICSD diagnosis (e.g., sleep-related cluster headache). Sleep-related headache is a purely descriptive diagnosis and is conferred when more than $75 \%$ of the headaches occur in relation to sleep; this diagnosis does not preclude the diagnosis of other headaches, or sleep disorders. For example, sleep-related headaches may include cluster headache, migraine and chronic paroxysmal hemicrania, morning headache secondary to sleep-disordered breathing or hypoxia, tension-type headache secondary to sleep bruxism, and others, provided that $75 \%$ of headaches occur in relation to sleep. Presumably, hypnic headache in ICHD-II can also be a classifiable condition among sleep-related headaches in $\operatorname{ICSD}[4,11,12]$.
The prevalence of headache in OSA has been reported to range from $15 \%$ to more than $50 \%$. There is some conflicting evidence about this association [13]. A few epidemiological studies have assessed the co-morbidity of various sleep and headache complaints. In a recent large European study of 18980 telephone interviews representing the populations of the United Kingdom, Germany, Spain, Portugal and Italy, the prevalence of 'chronic morning headache' (i.e., morning headache occurring 'daily', 'often' or 'sometimes') was reported at $7.6 \%$ with the incidence greater in females than males. Individuals with morning headache were more likely to exhibit insomnia, circadian rhythm disorder, loud snoring, sleep-related breathing disorder, nightmares and dyssomnia not otherwise specified than individuals without headache. Risk ratios increased for all sleep disorders except insomnia, when the data reanalysed in a model using only 'daily' morning headache [14]. In our study $45.4 \%$ of patients had specific sleep disturbances and $38.9 \%$ of them report- 
ed headache also. These patients showed a statistically significant degree of airway obstruction findings compared to those with no sleep disturbances. Excluding one patient with symptomatic cough headache, all subjects were diagnosed as primary headache subtypes. When it was combined with correlation and logistic regression analysis results, it could be hypothesised that the combination of headache and sleep disturbances was far from a coincidence. Apart from clinical variables, the levels of chronic hypoxaemia and hypercarbia had an important effect on specific types of sleep disturbances.

In the study of Ulfberg et al. [15], $18 \%$ of the patients with sleep apnoea or heavy snoring reported headache upon awakening 'often' or 'very often' compared with only $5 \%$ of the general population. Large epidemiologic studies show a statistically significant association between snoring or sleep apnoea and headaches [15, 16]. Detailed studies suggest that OAS in particular is associated with morning headache [17-19]. Göder et al [20]. reported that sleep disturbances, independent of their origin, lead to morning headache, which is further supported by Blau's study [21] concerning sleep deprivation headache. Headaches might be caused by a dysfunction of central pain-modulating circuits in regions such as the periaqueductal grey, the dorsolateral pontine tegmentum and the rostral ventromedial medulla $[22,23]$. The authors stated that alterations in brain regions that were equally involved in sleep regulation and nociception [24] contributed to a dysfunction of central pain-inhibitory activity, resulting in the occurrence of headache after awakening in vulnerable patients. Other hypotheses that were discussed were autonomic surges and blood pressure dysregulation associated with arousals, fragmented sleep and sleep stage alteration, increased intracranial pressure during apnoeas, daytime sleepiness or fatigue and changes in head and neck position with altered muscle tension [20]. At this point, as a clinical based study, we do not know which factor is most important. However, our data suggested the importance of additional effects of the combination of chronic hypoxaemia and sleep disturbances on headache complaints of COPD subjects. We also suggested that the effects of hypercarbia had been ignored in both headaches and sleep disturbances.
A large cross-sectional study of Danish middle-aged and elderly males identified a strong statistical relationship between snoring and non-specific headache (i.e., having any form of headache) [25]. Snoring is considered a sensitive though not a specific indicator of OSA in epidemiological research [4]. Our clinical based study suggested the important effect of snoring on headache (OR: 16.2). On the other hand, chronic airway obstruction parameters, hypoxia and hypercarbia showed important effects on snoring of COPD patients with headache (Table 3).

A high incidence of sleep complaints has been reported in studies of headache patients. Diagnostic sleep studies on 25 headache patients who complained of nocturnal or morning headache identified a sleep disorder sufficient to merit formal diagnosis in 13 patients, including OSA, periodic limb movements and fibrositis. In an expansion of this study, Paiva et al. [26] found that $17 \%$ of headache patients reported that their headaches were proximally related to sleep. Although polysomnographic records were not included, our study suggested supportive data of OSA and other sleep disturbances using pneumonic REST and structured interview. Statistical analysis also suggested significant association between headache and sleep disturbances with different scores.

Recently, the temporal relationship between sleep and head pain has been demonstrated with a prospective study. The application of time-series analysis with lagged correlations allowed Penzien and colleagues to examine temporal relationships between nightly sleep patterns and headache [27]. These findings demonstrate empirically that sleep patterns may play an important role in precipitating or exacerbating recurrent headache and support anecdotal observations regarding the temporal patterns of sleep and headache [4]. At this point we are not aware of the importance of drugs used for COPD (i.e., anticholinergics, theophylline etc.) to sleep disturbances or headache.

In conclusion, possibly being a specific subtype of elderly headache, headache in patients with moderate or severe COPD is a common problem especially in women and future studies are needed to obtain more knowledge about its pathophysiological and clinical basis.

\section{References}

1. Global Initiative for Chronic Obstructive Lung Disease (2003) Pocket guide to COPD diagnosis, management, and prevention. A guide for physicians and nurses. National Institutes of Health, National Heart, Lung and Blood Institute, Update
2. Headache Classification Committee of the International Headache Society (2004) The internal classification of headache disorders. Cephalalgia 24[Suppl 1]:1-150
3. Lisotto C, Mainardi F, Maggioni F, Zanchin G (2004) Episodic hypnic headache? Cephalalgia 24:681-685

4. Rains JC, Poceta S (2005) Sleep-related headache syndromes. Semin Neurol 25:69-80 
5. Weissman MM, Greenwald S, NinoMurcia G, Dement WC (1997) The morbidity of insomnia uncomplicated by psychiatric disorders. Gen Hosp Psychiatry 19:245-250

6. Ancoli-Israel S, Roth T (1999) Characteristics of insomnia in the United States: results of the 1991 National Sleep Foundation Survey. I. Sleep 22[Suppl 2]:S347-S353

7. Rasmussen BK, Olesen J (1992) Symptomatic and nonsymptomatic headaches in a general population. Neurology 42:1225-1231

8. Quanjer PH, Tammaling GJ, Cotes JE (1993) Lung volumes and forced respiratory flows: Report of the Working Party on Standardization of Lung Function Tests; European Community for Steel and Coal Official Statement of the European Respiratory Society. Eur Respir J 6[Suppl 16]:5-40

9. Ries AL (1989) Measurement of lung volumes. Clin Chest Med 10:177-186

10. Gardner RM, Crapo RO, Nelson SB (1989) Spirometry and flow-volume curves. Clin Chest Med 10:145-154

11. American Sleep Disorders Association Diagnostic Classification Steering Committee (1997) In: Thorpy M (ed.) The International Classification of Sleep Disorders - Revised: diagnostic and coding manual. Davies, Rochester, MN
12. Loh NK, Dinner DS, Foldvary N, Skobieranda F, Yew WW (1999) Do patients with obstructive sleep apnea wake up with headaches? Arch Intern Med 159:1765-1768

13. Idiman F, Oztura I, Baklan B, Ozturk V, Kursad F, Pakoz B (2004) Headache in sleep apnea syndrome. Headache 44:603-606

14. Ohayon MM (2004) Prevalence and risk factors of morning headaches in the general population. Arch Intern Med 164:97-102

15. Ulfberg J, Carter N, Talbäck M, Edling C (1996) Headache, snoring and sleep apnea. J Neurol 243:621-625

16. Jennum $P$, Hein HO, Suadicani $P$, Gyntelberg F (1994) Headache and cognitive dysfunctions in snorers. Arch Neurol 51:937-942

17. Guilleminault C, Van den Hoed J, Mittler MM (1978) Clinical overview of the sleep apnea syndromes. In: Guilleminault C, Dement WC (eds) Sleep apnea syndromes. Alan R. Liss, New York, NY, pp 1-12

18. Boutros NN (1989) Headache in sleep apnea. Tex Med 85:34-35

19. Loh NK, Dinner DS, Foldvary N et al (1999) Do patients with obstructive sleep apnea wake up with headaches? Arch Intern Med 159:1765-1768
20. Göder R, Friege L, Fritzer G, Strenge H, Aldenhoff JB, Hinze-Selch D (2003) Morning headaches in patients with sleep disorders: a systematic polysomnographic study. Sleep Med 4:385-391

21. Blau JN (1990) Sleep deprivation headache. Cephalalgia 10:157-160

22. Fields HL (1997) Pain modulation and headache. In: Goadsby PJ, Silberstein SD (eds) Headache. ButterworthHeinemann, Newton, MA, pp 39-58

23. Fields HL (2000) Pain modulation: expectation, opioid analgesia and virtual pain. Prog Brain Res 122:245-252

24. Ksatri AM, Baghdoyan HA, Lydic R (1998) Cholinomimetics, but not morphine, increase antinociceptive behaviour from pontine reticular regions regulating rapid-eye-movement sleep. Sleep 21:677-685

25. Jennum P, Sjol A (1994) Self-assessed cognitive function in snorers and sleep apneics - an epidemiological study of 1,504 females and males aged 30-60 years: the Dan-MONICA II Study. Eur Neurol 34:204-208

26. Paiva T, Farinha A, Martins A, Batista A, Guilleminault C (1997) Chronic headaches and sleep disorders. Arch Intern Med 157:1701-1705

27. Penzien DB, Rains JC, Andrew ME, Galovski T, Mohammad Y, Mosely T (2001) Relationship of daily stress, sleep, and headache: a time series analysis. Cephalalgia 21:262-263 